\title{
MANEJO DE PACIENTES COM SÍNDROME DO ANTICORPO ANTIFOSFOLÍPIDE
}

\section{ARTIGO DE REVISÃO}

MORATO, Luiza Elias' ${ }^{1}$, CASTRO, Fernando Mendes², SOUZA FILHO, Francisco Carlos Moraes ${ }^{3}$, GARCIA, Hanna Michelle Melo4, PIEROTE, lasmin Moraes ${ }^{5}$, SCHINCARIOL, Isadora Bossolan ${ }^{6}$, ARAUJO, João Marcos Batista Gomes De ${ }^{7}$, SANTOS, Juan Carlos Oliveira ${ }^{8}$, DRUMOND, Karolayne Cecília Pinto ${ }^{9}$, OLIVEIRA,

${ }^{1}$ Médica graduada pela Faculdade de Medicina de Barbacena.

${ }^{2}$ Discente do curso de Medicina do Centro Universitário UNINOVAFAPI.

${ }^{3}$ Discente do curso de Medicina do Centro Universitário UNINOVAFAPI.

${ }^{4}$ Discente do curso de Medicina da Universidade UNICEUMA.

${ }^{5}$ Discente do curso de Medicina da UNIFACID.

${ }^{6}$ Discente do curso de Medicina do Centro Universitário Max Planck - UniMAX.

${ }^{7}$ Discente do curso de Medicina da Faculdade Santa Maria.

${ }^{8}$ Discente do curso de Medicina da UNIFACID.

${ }^{9}$ Discente do curso de Medicina da Faculdade da Saúde e Ecologia Humana.

$\mathrm{RC}: 88466$

Disponível em: https://www.nucleodoconhecimento.com.br/saude/anticorpo-antifosfolipide 
Igor de Sousa ${ }^{10}$, RIBEIRO, Yana Sarah Fernandes Souza ${ }^{11}$, VASCONCELOS, Gilberto Loiola de ${ }^{12}$

MORATO, Luiza Elias. Et al. Manejo de pacientes com síndrome do anticorpo antifosfolípide. Revista Científica Multidisciplinar Núcleo do Conhecimento. Ano 06, Ed. 06, Vol. 07, pp. 169-181. Junho de 2021. ISSN: 2448-0959, Link de acesso: https://www.nucleodoconhecimento.com.br/saude/anticorpo-antifosfolipide,

DOI: 10.32749/nucleodoconhecimento.com.br/saude/anticorpo-antifosfolipide

\section{RESUMO}

Objetivo: Este estudo objetiva clarificar evidências científicas relacionadas ao manejo clínico adequado de pacientes portadores de Síndrome do Anticorpo Antifosfolípide (SAAF). Métodos: $O$ trabalho configura uma revisão bibliográfica integrativa, quantiqualitativa, de natureza aplicada, objetivo descritivo e procedimento bibliográfico. Utilizou-se a base de dados PUBMED e sites aliados e os Descritores em Ciências da Saúde, nos quais foram triados 12 artigos para análise, disponíveis no recorte temporal de cinco anos e publicados nos idiomas espanhol, inglês e português. Resultados: Dentre os 7 estudos analisados, $28,5 \%$ desenvolveram 0 comparativo entre fármacos disponíveis para uso no manejo de pacientes portadores de Síndrome do Anticorpo Antifosfolípide (SAAF). Rivaroxabana, quando comparada à Varfarina, não precipitou risco maior de eventos trombóticos, o que atestou que a droga pode ser utilizada com segurança e eficácia em pacientes portadores de SAAF

10 Discente do curso de Medicina da Universidade Federal de Campina Grande, Centro de Formação dos Professores, Cajazeiras - Paraíba.

11 Discente do curso de Medicina do Centro Universitário INTA - UNINTA.

12 Orientador. Médico residente em Clínica Médica, formado pela Universidade Federal do Ceará.

RC: 88466

Disponível em: https://www.nucleodoconhecimento.com.br/saude/anticorpo-antifosfolipide 
que resguardam histórico prévio de tromboembolismo venoso. De maneira oposta, em pacientes com SAAF de alto risco trombótico foi evidenciado melhor desfecho com o uso de inibidores da vitamina K. Conclusão: Observou-se que o diagnóstico precoce de SAAF tem função significativa no desenvolvimento dos desfechos clínicos. Faz-se necessária, ainda, uma abordagem individualizada dos pacientes, com o fito de compreender as nuances das comorbidades associadas à síndrome.

Palavras-chave: Síndrome Antifosfolipídica, Diagnóstico, Ação Terapêutica.

\section{INTRODUÇÃO}

A Síndrome Antifosfolipídica (SAF) é caracterizada como uma patologia autoimune, com predomínio de eventos trombóticos venosos e/ou arteriais e morbidade gestacional. Além disso, pode apresentar ainda manifestações como trombocitopenia, doenças valvulares, nefropatias e distúrbios neurológicos. De fato, a doença é marcada pela presença de anticorpos antifosfolipídeos, como anti-beta2-glicoproteína I e anticoagulante lúpico, podendo ser classificada primária ou secundária a outras doenças, com destaque para o Lúpus Eritematoso Sistêmico (LES) (PALLI; KRAVVARITI; TEKTONIDOU, 2019).

Estudo feito por Costa (2019) evidenciou um perfil epidemiológico no qual 93,9\% dos portadores de SAF eram do sexo feminino. Neste perfil, foram observados óbitos de fetos de mais de 10 semanas de idade gestacional, morfologicamente normais, em $62,16 \%$ das gestações, bem como abortos espontâneos em 18,92\% das mulheres grávidas portadoras da síndrome. Notou-se, ainda, 18,92\% de incidência de partos prematuros com menos de 34 semanas, associados à pré-eclâmpsia e insuficiência placentária.

As principais repercussões clínicas da SAF se relacionam aos efeitos tromboembólicos, os quais podem afetar os mais diversos órgãos e sistemas e precipitar, a título de exemplo, infarto do fígado, acidente vascular cerebral, tromboembolismo e hipertensão pulmonar, trombose da artéria e veia renal e

RC: 88466

Disponível em: https://www.nucleodoconhecimento.com.br/saude/anticorpo-antifosfolipide 
trombose da veia e artéria da retina. Nesse sentido, a insuficiência placentária pode também estar relacionada à perda da circulação útero-placentária pelos mesmos eventos, sendo esta a maior causa de perda fetal tardia na síndrome, geralmente a partir do segundo trimestre (LOPES et al., 2017).

Cumpre ressaltar, portanto, que a SAF é uma comorbidade que exige diagnóstico precoce e, principalmente, manejo adequado. A despeito de ser considerada uma doença rara (incidência estimada de 5 casos por 100.000 pessoas por ano), a síndrome afeta principalmente pessoas jovens em idade reprodutiva, causando sequelas físicas e psicológicas. Logo, o diagnóstico acurado e a abordagem correta desta síndrome melhoram consideravelmente a qualidade de vida, o prognóstico e, muitas vezes, permitem o diagnóstico de outras doenças autoimunes que podem estar relacionadas com a patologia, o que propicia a possibilidade de melhora da situação global do paciente (COSTA, 2019).

O objetivo do estudo é evidenciar quais seriam as melhores propostas de intervenção para pacientes portadores da síndrome, uma vez que um manejo adequado torna o desfecho mais favorável para estes indivíduos.

\section{METODOLOGIA}

Este trabalho consiste em uma revisão bibliográfica do tipo integrativa com abordagem quanti-qualitativa, de natureza aplicada, objetivo descritivo e procedimento bibliográfico.

Para sua elaboração, durante o mês de março de 2021 foi realizada uma pesquisa por meio da base de dados PUBMED e sites aliados. Inicialmente, para a escolha dos descritores, utilizou-se da ferramenta Descritores em Ciências da Saúde (DeCS). Dessa forma, os descritores escolhidos foram: "síndrome antifosfolipídica", "diagnóstico" e "ação terapêutica" nos idiomas português, inglês e espanhol, tendo sido utilizados, também, sinônimos pertinentes para tais descritores. Além disso, os 
operadores booleanos parênteses, $A N D$ e $O R$ foram utilizados para formatação da fórmula de busca.

Dando prosseguimento à pesquisa, iniciou-se a seleção dos artigos e, para isso, foram empregados os seguintes filtros: recorte temporal de cinco anos e filtros com o idioma espanhol, inglês e português, nesta ordem. Dentro destes critérios, 12 artigos foram extraídos do PUBMED.

No que tange aos critérios de elegibilidade, os artigos selecionados foram aqueles que incluíram pacientes, crianças ou adultos, que apresentam síndrome antifosfolipídica, bem como estudos que abordam os principais métodos diagnósticos e terapêuticos para tal doença, inclusas no recorte temporal pré-determinado, independente da etnia ou sexo do paciente. Foram excluídos os artigos que abordavam outros pontos da síndrome, além do diagnóstico e da terapêutica. Excluiu-se, também, artigos de opinião, metanálises, revisões sistemáticas, estudos duplicados e artigos que não estavam disponíveis na íntegra.

Em seguida, dois pesquisadores, ISO e KCPD, avaliaram todos os títulos e resumos dos artigos e selecionaram os estudos baseados nos critérios de elegibilidade previamente citados. A partir dessa análise e conectando aos nossos objetivos, observou-se que 7 desses artigos estavam aptos a serem analisados no trabalho.

Salienta-se que não foi necessária a utilização do comitê de ética neste trabalho, pois trata-se de uma revisão bibliográfica que não possui aplicação prática.

Por fim, após a análise dos dados, o resultado foi disposto em um quadro com aspectos técnicos dos artigos, contendo as seguintes informações: título, ano e local de publicação, autoria e principais resultados.

$\mathrm{RC}: 88466$

Disponível em: https://www.nucleodoconhecimento.com.br/saude/anticorpo-antifosfolipide 


\section{RESULTADOS}

Quadro 1: Principais Desfechos dos Artigos Analisados

\begin{tabular}{|c|c|c|c|}
\hline & Título & $\begin{array}{l}\text { Autores, ano } \\
\text { de } \\
\text { publicação e } \\
\text { país de } \\
\text { origem }\end{array}$ & Principais Resultados \\
\hline $\begin{array}{l}\text { Artigo } \\
\text { I }\end{array}$ & $\begin{array}{l}\text { Rivaroxaban vs warfarin in } \\
\text { high-risk patients with } \\
\text { antiphospholipid } \\
\text { syndrome }\end{array}$ & $\begin{array}{l}\text { Pengo et al., } \\
2018, \\
\text { Estados } \\
\text { Unidos }\end{array}$ & $\begin{array}{l}\text { Rivaroxaban foi considerada } \\
\text { inferior a Warfarin, por } \\
\text { apresentar riscos elevados em } \\
\text { comparação aos benefícios. O } \\
\text { estudo foi interrompido } \\
\text { precocemente devido a } \\
\text { eventos tromboembólicos } \\
\text { arteriais no seguimento com a } \\
\text { Rivaroxaban, } \\
\text { nenhum evento desta natureza } \\
\text { foi registrado com a utilização } \\
\text { de Warfarin. }\end{array}$ \\
\hline $\begin{array}{l}\text { Artigo } \\
\text { II }\end{array}$ & $\begin{array}{l}\text { Rivaroxaban versus } \\
\text { warfarin to treat patients } \\
\text { with thrombotic } \\
\text { antiphospholipid } \\
\text { syndrome, with or without } \\
\text { systemic lupus } \\
\text { erythematosus (RAPS): a } \\
\text { randomised, controlled, }\end{array}$ & $\begin{array}{l}\text { Cohen et al., } \\
\text { 2016, Reino } \\
\text { Unido }\end{array}$ & $\begin{array}{l}\text { O risco de eventos trombóticos } \\
\text { com Rivaroxaban não foi maior } \\
\text { do que com a Warfarin. A } \\
\text { Rivaroxaban apresentou-se } \\
\text { como uma alternativa segura e } \\
\text { eficaz, com a vantagem de não } \\
\text { precisar de monitorização } \\
\text { contínua da anticoagulação. }\end{array}$ \\
\hline
\end{tabular}

RC: 88466

Disponível em: https://www.nucleodoconhecimento.com.br/saude/anticorpo-antifosfolipide 


\begin{tabular}{|c|c|c|c|}
\hline & $\begin{array}{l}\text { en-label, phase } 2 / 3 \text {, } \\
\text { n-inferiority trial. }\end{array}$ & & \\
\hline $\begin{array}{l}\text { Artigo } \\
\text { III }\end{array}$ & $\begin{array}{l}\text { Apixaban for the } \\
\text { Secondary Prevention of } \\
\text { Thrombosis Among } \\
\text { Patients } \quad \text { With } \\
\text { Antiphospholipid } \\
\text { Syndrome: } \\
\text { Rationale and Design } \\
\text { (ASTRO-APS). }\end{array}$ & $\begin{array}{l}\text { Woller et al., } \\
2016, \\
\text { Estados } \\
\text { Unidos }\end{array}$ & $\begin{array}{l}\text { No estudo prospectivo a ser } \\
\text { realizado, espera-se provar } \\
\text { que a Apixaban será segura e } \\
\text { eficaz na prevenção de } \\
\text { trombose recorrente em } \\
\text { pacientes com a Síndrome do } \\
\text { Anticorpo Antifosfolípide. }\end{array}$ \\
\hline Artigo & $\begin{array}{l}\text { Omega-3 Fatty Acid } \\
\text { Supplementation } \\
\text { Improves Endothelial } \\
\text { Function in Primary } \\
\text { Antiphospholipid } \\
\text { Syndrome: A Small-Scale } \\
\text { Randomized Double-Blind } \\
\text { Placebo-Controlled Trial. }\end{array}$ & $\begin{array}{l}\text { Felau et al., } \\
\text { 2018, Suíça }\end{array}$ & $\begin{array}{l}\text { A suplementação do Ômega-3 } \\
\text { ajudou na melhora da função } \\
\text { endotelial em pacientes com a } \\
\text { Síndrome do Anticorpo } \\
\text { Antifosfolípide Primária bem } \\
\text { controlada e, portanto, foi } \\
\text { considerada uma droga } \\
\text { segura, com capacidade de } \\
\text { reduzir risco cardiovascular. }\end{array}$ \\
\hline $\begin{array}{l}\text { Artig } \\
\mathrm{V}\end{array}$ & $\begin{array}{l}\text { Protein } \\
\text { isomerase isulfide } \\
\text { blocks } \\
\text { generation in humans by } \\
\text { interfering with platelet } \\
\text { factor V activation }\end{array}$ & $\begin{array}{l}\text { Stopa et al., } \\
2017, \\
\text { Estados } \\
\text { Unidos }\end{array}$ & $\begin{array}{l}\text { A isoquercetin oral atuou como } \\
\text { inibidora da proteína } \\
\text { dissulfeto-isomerase, } \\
\text { contribuindo para redução da } \\
\text { geração de plaquetas } \\
\text { trombina-dependentes. Dessa } \\
\text { forma, uma nova classe de } \\
\text { medicamento atuando nesta } \\
\text { via pode ser desenvolvida. }\end{array}$ \\
\hline
\end{tabular}




\begin{tabular}{|c|c|c|c|}
\hline $\begin{array}{l}\text { Artigo } \\
\text { VI }\end{array}$ & $\begin{array}{l}\text { Treatment of the } \\
\text { antiphospholipid } \\
\text { syndrome with direct oral } \\
\text { anticoagulants. }\end{array}$ & $\begin{array}{l}\text { Bauersachs } \\
\text { et al., 2019, } \\
\text { Suíça }\end{array}$ & $\begin{array}{l}\text { Pacientes com Lúpus podem } \\
\text { ser tratados com } \\
\text { anticoagulantes orais caso } \\
\text { tenham sofrido eventos } \\
\text { venosos anteriores. Pacientes } \\
\text { com SAAF de alto risco ou com } \\
\text { eventos arteriais anteriores } \\
\text { devem receber antagonistas } \\
\text { da vitamina } \mathrm{K} \text { (Warfarin). }\end{array}$ \\
\hline $\begin{array}{l}\text { Artigo } \\
\text { VII }\end{array}$ & $\begin{array}{l}\text { Type I Interferon Signature } \\
\text { in Primary } \\
\text { Antiphospholipid } \\
\text { Syndrome: Clinical and } \\
\text { Laboratory Associations. }\end{array}$ & $\begin{array}{l}\text { Palli, } \\
\text { Kravvariti e } \\
\text { Tektonidou, } \\
\text { 2019, Suiça }\end{array}$ & $\begin{array}{l}\text { Pacientes com Síndrome do } \\
\text { Anticorpo Antifosfolípide } \\
\text { Primária, principalmente } \\
\text { naqueles com anticorpos } \\
\text { B2GPI, possuem um alto nível } \\
\text { de Interferon tipo } 1 \text {, sendo } \\
\text { necessários mais estudos para } \\
\text { o entendimento da patogenia } \\
\text { das complicações trombóticas } \\
\text { e não trombóticas. }\end{array}$ \\
\hline
\end{tabular}

Fonte: Autoria Própria, 2021.

Dentre os 7 estudos analisados, 28,5\% ( $n=2)$ estabeleceram parâmetros comparativos entre fármacos disponíveis para uso no manejo de pacientes portadores de Síndrome do Anticorpo Antifosfolípide (SAAF). Neste sentido, Pengo et al. (2018) estabeleceram um comparativo entre o uso de Rivaroxabana e Varfarina entre pacientes portadores de SAAF com o padrão triplo-positivo para os anticorpos anticoagulante lúpico, anti-cardiolipina e anti- $\beta 2$-glicoproteína I. O trabalho identificou que Rivaroxabana, fármaco inibidor direto do fator $\mathrm{Xa}$, não exerce efeito protetor de eventos tromboembólicos em pacientes de alto risco, tendo sido observados quatro acidentes vasculares encefálicos isquêmicos e três infartos agudos do miocárdio

RC: 88466

Disponível em: https://www.nucleodoconhecimento.com.br/saude/anticorpo-antifosfolipide 
dentre os participantes do estudo que fizeram uso do medicamento. Isto foi atribuído à exposição a níveis plasmáticos inadequados da droga, má aderência medicamentosa e mecanismo de ação diferente entre os anticoagulantes.

Vale salientar que, por seu caráter trombofílico e de frequente associação com outras doenças autoimunes, a SAAF se impõe como um grande desafio no que tange ao manejo de características clínicas e complicações. Neste contexto, observou-se que pacientes portadores de apenas um ou dois dos anticorpos antifosfolipídicos (exceto anticorpo anticoagulante lúpico), por configurarem perfis de baixo risco para trombose, podem ser tratados adequadamente com anticoagulantes orais diretos (rivaroxaban, apixaban, dabigatran e edoxaban). Por sua vez, pacientes com SAAF de alto risco trombótico (com presença de três dos anticorpos antifosfolípides ou com histórico de doença arterial prévia) não devem ser tratados com tais fármacos mas, sim, com inibidores da vitamina K. Cabe ressaltar que em tais indivíduos em uso de Varfarina deve-se objetivar um INR-alvo de 2 a 3 . Permanece controverso, no entanto, o benefício do uso de ácido acetilsalicílico em associação à Varfarina no contexto de pacientes com eventos arteriais trombóticos antecedentes (BAUERSACHS et al., 2019).

Em contrapartida, estudo britânico identificou que o uso de Rivaroxabana, quando comparado à Varfarina, não precipitou risco maior de eventos trombóticos, o que atestou que a droga pode ser utilizada com segurança e eficácia em pacientes portadores de SAAF que resguardam histórico prévio de tromboembolismo venoso. O estudo avaliou indivíduos que já faziam uso de Varfarina como prevenção secundária de acometimentos venosos tromboembólicos, tendo sido analisados o potencial endógeno de trombina e o pico de trombina dentre os participantes do ensaio. Percebeu-se, portanto, que pacientes que fizeram uso de Rivaroxabana desenvolveram taxas mais altas de potencial de trombina, enquanto que os picos séricos de geração de trombina, por sua vez, foram mais altos em pacientes que permaneceram utilizando Varfarina (COHEN et al., 2016). 
Sabe-se que a disfunção endotelial tem um papel importante na patogênese da SAAF, uma vez que predispõe a ocorrência de eventos trombóticos e tromboembólicos, como acidente vascular encefálico e infarto agudo do miocárdio. À vista disto, um dos estudos demonstrou que a suplementação de n-3 PUFA (Ômega-3) foi capaz de promover melhora da função endotelial em vários contextos. Desta forma, o ensaio clínico avaliou, durante 16 semanas, a eficácia da suplementação em pacientes com SAAF mediante a avaliação de 22 mulheres adultas portadoras da síndrome primária. Por fim, foram comprovadas a eficácia e a segurança do fármaco enquanto redutor do risco cardiovascular de tais pacientes (FELAU et al., 2018).

Cumpre salientar que a proteína dissulfeto-isomerase (PDI) é necessária para a agregação plaquetária e a formação de fibrina em cenários de lesão vascular ou inflamação. Deste modo, estudo norte-americano de Stopa et al. (2017) avaliou os efeitos da Isoquercetina, fármaco inibidor da PDI, em relação à geração de trombina, tanto em pacientes saudáveis quanto em pacientes com anticorpos antifosfolipídios elevados. Observou-se que a administração oral de Isoquercetina inibe a atividade da PDI no plasma, diminuindo, por consequência, a aglomeração plaquetária e a formação de fibrina, mediante o bloqueio do cofator enzimático Va. Constata-se, então, uma nova possibilidade de manejo terapêutico a ser explorada.

No que tange às abordagens imunoterapêuticas, um dos estudos objetivou analisar, em pacientes com SAAF primária, a expressão de Interferon I (IFN-I), citocina já descrita em células sanguíneas e teciduais de pacientes com LES e outras moléstias reumáticas. Neste sentido, identificou-se que pacientes acometidos pela SAAF detêm níveis mais elevados de IFN-I, bem como de anticorpos anti-ß2-glicoproteína I, proteína plasmática responsável pela inibição do fator XII da cascata de coagulação, bem como pela conversão de protrombina em trombina. Entretanto, faz-se necessária uma complementação dos estudos, para que sejam correlacionados os achados à patogenia das complicações tromboticas e não tromboticas (PALLI; KRAVVARITI e TEKTONIDOU, 2019).

$\mathrm{RC}: 88466$

Disponível em: https://www.nucleodoconhecimento.com.br/saude/anticorpo-antifosfolipide 
Neste cenário, têm sido desenvolvidas novas perspectivas de abordagem de pacientes portadores de SAAF. Dentre as quais, pode-se citar estudo piloto norteamericano de Woller et al. (2016), que objetiva analisar, através de pesquisa prospectiva e randomizada, a implantação de Apixaban como fármaco anticoagulante adjuvante ao tratamento de indivíduos portadores da Síndrome do Anticorpo Antifosfolípide. Nesse sentido, o trabalho tem por desfecho esperado a comprovação da segurança e eficácia do Apixaban no contexto de prevenção secundária de trombose, com a importante vantagem de prescindir da monitorização rigorosa do coagulograma.

\section{DISCUSSÃO}

\section{APRESENTAÇÃO CLÍNICA}

Como evidenciado em resultados, a SAAF possui suas manifestações clínicas predominantemente voltadas para eventos trombóticos venosos e/ou arteriais. Entre tais manifestações, apresenta-se como a mais comum, ocorrendo em $32 \%$ dos pacientes diagnosticados com a síndrome, a trombose venosa profunda dos membros inferiores, morbidade que pode precipitar o quadro de embolia pulmonar. Abrangendo o segundo lugar de manifestação trombótica mais comum na SAAF, tem-se o Acidente Vascular Cerebral (AVC), dispondo, assim, do risco de acometer qualquer território encefálico, resultando em sequelas que comprometem a qualidade de vida do paciente (MARTINS et al., 2015).

De fato, a SAAF pode acometer diversos sistemas, incluindo sistema hepático, sistema nervoso central, sistema hematológico, sistema renal e sistema cardíaco. Ademais, manifestações obstétricas também podem estar envolvidas, podendo ter como consequências: pré-eclâmpsia, insuficiência placentária, crescimento intrauterino restrito $(\mathrm{CIR})$, parto pré-termo e até mesmo morte fetal. Além disso, a SAAF pode ser considerada como o principal precedente de abortamento habitual, estando envolvida em 3 a 15\% dos casos (MONTENEGRO; REZENDE, 2014).

$\mathrm{RC}: 88466$

Disponível em: https://www.nucleodoconhecimento.com.br/saude/anticorpo-antifosfolipide 
As manifestações cutâneas são de grande importância para o diagnóstico de um paciente com suspeita de SAAF, como atesta estudo proposto por Santamaria et al. (2005). Neste, analisou-se que $41 \%$ dos pacientes diagnosticados com a síndrome possuem os sinais cutâneos como primeiro indício. Ademais, as lesões dermatológicas são consequências da oclusão vascular, tendo havendo o livedo reticular como um sinal iterado, constituído por reação cutânea cianótica que apresenta aspecto rendilhado. Além disso, por tratar-se de livedo reticular secundário à SAAF, é caracterizado por trama reticulada cutânea de rede interrompida e assimétrica, denominada livedo recemoso. Vale salientar que outras manifestações dermatológicas também podem ser associadas à SAAF, quais sejam: acrocianose, ulcerações cutâneas, necroses cutâneas, síndrome dos dedos azuis, tromboflebite, isquemia ou grangrena digital, hemorragias lineares subungueais, entre outras (RIVITTI, 2014).

\section{MANEJO}

Como já exposto anteriormente, a SAAF representa um grande desafio clínico, pois está associada a risco trombótico muito elevado. No que concerne à abordagem terapêutica, atualmente os antagonistas de vitamina $\mathrm{K}$, a exemplo da warfarin, configuram o padrão atual de tratamento anticoagulante.

Percebe-se, no entanto, um crescente interesse pela investigação da adoção de fármacos como a rivaroxaban (anticoagulante oral direto), que possui relação riscobenefício duvidosa, visto que provocou muitos efeitos tromboembólicos antes mesmo do fim do estudo realizado por Pendo et al. (2018). Em contrapartida, o estudo de Cohen et al. (2016) revelou que tais medicamentos possuem riscos semelhantes aos antagonistas da vitamina $\mathrm{K}$, inclusive em comparação a heparinas de baixo peso molecular.

De acordo com estudo realizado por Palli; Kravvariti e Tektonidou (2019), pacientes com SAAF primária, principalmente aqueles com anticorpos $\beta 2 \mathrm{GPI}$, possuem alto

RC: 88466

Disponível em: https://www.nucleodoconhecimento.com.br/saude/anticorpo-antifosfolipide 
nível de interferon tipo 1. Nesse contexto, foram realizados estudos prospectivos, como o de Woller et al. (2016), que defendem a eficácia e a segurança do apixaban, fármaco inibidor direto do fator $\mathrm{Xa}$, na prevenção de trombose recorrente em pacientes com SAAF. Além disso, Stopa et al. (2017) identificaram que a isoquercetin oral atua como inibidora da proteína dissulfeto-isomerase, contribuindo para redução da geração de plaquetas trombina-dependentes e despertando, assim, a possibilidade de intervenção farmacológica nesta via. Tais estudos, no entanto, embora promissores, ainda não configuram alternativas viáveis às terapias existentes até o momento.

Ademais, acredita-se que as células endoteliais desempenhem um papel central na patogênese da síndrome, uma vez que ocorre uma disfunção endotelial provocada pelo aumento da expressão de moléculas de adesão e da secreção de citocinas inflamatórias, como interleucina-1 e fator de necrose tumoral. Nesse sentido, Felau et al. (2018) elaborou estudo defendendo o papel da suplementação com ômega 3 na melhora da função endotelial em pacientes com SAAF primária bem controlada, sendo considerada uma droga segura e que diminui o risco cardiovascular, em consonância com estudo realizado por Cerqueira (2017).

Outrossim, no lúpus eritematoso sistêmico (LES) os anticorpos antifosfolípides estão presentes em mais de um terço dos pacientes, porém nem todos apresentam a síndrome clínica e, quando desenvolvem, fazem parte da síndrome antifosfolípide secundária (FREITAS et al., 2003). Dessa maneira, há um consenso entre as literaturas de Bauersachs et al. (2019) e Santamaria et al. (2005) de que o tratamento de pessoas com LES pode ser feito com anticoagulantes orais caso tenham sofrido eventos venosos anteriores e pacientes com SAF de alto risco ou com eventos arteriais anteriores devem receber antagonistas da vitamina $\mathrm{K}$ (Warfarin).

RC: 88466

Disponível em: https://www.nucleodoconhecimento.com.br/saude/anticorpo-antifosfolipide 


\section{CONCLUSÃO}

Clarifica-se, portanto, a importância de compreender adequadamente a SAAF, isto é, estabelecer suas principais manifestações sistêmicas, seu perfil epidemiológico e suas expressões clínicas. Ademais, nota-se evidente que o diagnóstico feito precocemente colabora para um bom desfecho clínico direcionado ao paciente.

Em relação ao diagnóstico do paciente com SAAF, faz-se valoroso ser realizado precocemente, com o fito de propiciar um melhor e mais acurado manejo terapêutico. As principais drogas com demonstrada eficácia para o tratamento da SAAF ainda são os antagonistas da Vitamina $\mathrm{K}$, mas anticoagulantes orais diretos também são utilizados no tratamento.

Isso posto, constata-se que faz-se necessário o desenvolvimento de novos estudos. Esse fato esclarece-se por que ainda não existe um satisfatório desfecho de consenso no tratamento e manejo da SAAF.

\section{REFERÊNCIAS}

BAUERSACHS, Rupert et al. Treatment of the antiphospholipid syndrome with direct oral anticoagulants. VASA. Zeitschrift für Gefässkrankheiten., Suíça, 2019.

CERQUEIRA, Sheylla Maryelleen Felau. Eficácia e segurança da suplementação de ômega 3 em pacientes com a síndrome do anticorpo antifosfolípide primário. 2017. Dissertação (Mestrado em Processos Imunes e Infecciosos) - Faculdade de Medicina, University of São Paulo, São Paulo, 2017. Disponúvel em: doi:10.11606/D.5.2018.tde-20022018-115936. Acesso em: 13 de abril de 2021.

COHEN, Hannah et al. Rivaroxaban versus warfarin to treat patients with thrombotic antiphospholipid syndrome, with or without systemic lupus erythematosus (RAPS): a randomised, controlled, open-label, phase $2 / 3$, non-inferiority trial. The Lancet, Haematology, Reino Unido, v. 3, 2016.

$\mathrm{RC}: 88466$

Disponível em: https://www.nucleodoconhecimento.com.br/saude/anticorpo-antifosfolipide 
COSTA, R. H. A. Perfil Epidemiológico dos Pacientes com Síndrome do Anticorpo Antisfosfolípide. Monografia (Programa de Residência em Reumatologia) - Hospital Geral de Fortaleza. Fortaleza - CE. 2019. Disponível em: <http://extranet.hgf.ce.gov.br/jspui/bitstream/123456789/485/1/PERFIL\%20EPIDEMI OL\%c3\%93GICO\%20DOS\%20PACIENTES\%20COM\%20S\%c3\%8dNDROME\%20D O\%20ANTICORPO\%20ANTIFOSFOL\%c3\%8dPIDE\%20MONOGRAFIA\%20RAFAE L\%20COSTA.pdf>. Acesso em: 26 março 2021.

FELAU, Sheylla M et al. Omega-3 Fatty Acid Supplementation Improves Endothelial Function in Primary Antiphospholipid Syndrome: A Small-Scale Randomized DoubleBlind Placebo-Controlled Trial. Frontiers in Immunology, Suiça, v. 9, 2 mar. 2018.

FREITAS, Max Victor Carioca et al. Síndrome do anticorpo antifosfolípide: estudo comparativo das formas primária e secundária. Rev. Bras. Reumatol., São Paulo, v. 43, n. 3, p. 153-159, June 2003. Disponível em: <http://www.scielo.br/scielo.php?script=sci_arttext\&pid=S048250042003000300005\&lng=en\&nrm=iso >. Acesso em: 13 abril 2021.

LOPES, Michelle Remião Ugolini et al. Atualização sobre a síndrome do anticorpo antifosfolipídeo. Rev. Assoc. Med. Bras., São Paulo, v. 63, n. 11, pág. 994-999, novembro de 2017.2 Disponível em: <http://www.scielo.br/scielo.php?script=sci_arttext\&pid=S0104$42302017001100994 \& \operatorname{lng}=e n \& n r m=i s o>$. Acesso em: 26 março 2021.

PALLI, Eleni; KRAVVARITI, Evrydiki; TEKTONIDOU, Maria G. Type I Interferon Signature in Primary Antiphospholipid Syndrome: Clinical and Laboratory Associations. Frontiers in Immunology, Suiça, v. 10, 15 mar. 2019.

PENGO, Vittorio et al. Rivaroxaban vs warfarin in high-risk patients with antiphospholipid syndrome. Blood, Estados Unidos, v. 132, 27 set. 2018. 
SANTAMARIA, Jesus Rodriguez et al. Síndrome antifosfolípide. An. Bras. Dermatol., Rio de Janeiro, v. 80, n. 3, p. 225-239, June 2005. Disponível em: $<$ http://www.scielo.br/scielo.php?script=sci_arttext\&pid=S036505962005000300002\&lng=en\&nrm=iso >. Acesso em: 13 abril 2021

STOPA, Jack D et al. Protein disulfide isomerase inhibition blocks thrombin generation in humans by interfering with platelet factor $V$ activation. Journal of clinical investigation insight, Estados Unidos, 12 jan. 2017.

WOLLER, Scott C et al. Apixaban for the Secondary Prevention of Thrombosis Among Patients With Antiphospholipid Syndrome: Study Rationale and Design (ASTRO-APS). Clinical and Applied Thrombosis/Hemostasis, Estados Unidos, v. 22, 2016.

Enviado: Maio, 2021.

Aprovado: Junho, 2021. 